\title{
Inhibitory Activities of Platelet-Rich and Platelet-Poor Plasma on the Growth of Pathogenic Bacteria
}

\author{
Omid Maghsoudi ${ }^{1}$, Reza Ranjbar ${ }^{2}$, Seyyed Hesamoddin Mirjalili', Mahdi Fasihi-Ramandi ${ }^{2}$ \\ 1. Faculty of Veterinary Medicine, Islamic Azad University, Karaj Branch, Iran \\ 2. Molecular Biology Research Center, Baqiyatallah University of Medical Science, Tehran, Iran
}

\begin{tabular}{l} 
KEYWORDS \\
\hline Platelet Rich Plasma \\
Antibacterial effect \\
Infection \\
MIC \\
Agar-Well Diffusion
\end{tabular}

Article Info

Received 12 Oct 2015;

Accepted 11 Apr 2016;

Published Online 30 Aug 2016;

\section{ABSTRACT}

Background: The utility and efficacy of novel materials in tissue regeneration and antimicrobial therapy are contingent upon the employment of either blood derivatives rich in platelets or platelet-poor-plasma (PPP). This effect is largely mediated by the increased or decreased concentration of platelets in the plasma. The current study aimed to analyze and evaluate the impact of platelet-rich (PRP) or PPP on inhibiting the growth of human pathogenic bacteria and compare their effects with those of chloramphenicol and penicillin.

Methods: In the current comparative study, PRP-1 was generated using 1-step blood centrifugation method; whereas, for PRP-2 and PPP the 2-step centrifugation protocol was used. The antimicrobial activity of PRP-1, 2, and PPP were tested on Staphylococcus aureus, Escherichia coli, Klebsiella pneumonia, Pseudomonas aeruginosa, Streptococcus agalactiae, Staphylococcus epidermidis, Shigella sp. and Serratia sp. Well diffusion and serial micro-dilution methods were used for this purpose. Chloramphenicol and penicillin susceptibility were tested using the disk diffusion method.

Results: While whole blood (WB) and PPP had no discernible impact on the growth parameters of any of the bacteria tested in the current study, PRP-1 reduced the growth rate of a few selected strains. In addition, while PRP-2 clearly inhibited the growth of Shigella sp., E. coli, S. aureus, S. agalactiae, and S. epidermidis, it had no impact on the growth of K. pneumoniae, P. aeruginosa, and Serratia sp

Conclusion: It can be claimed that there is a strong correlation between the concentration of platelets and the antibacterial activity of PRP.

Corresponding Information: Dr Mahdi Fasihi-Ramandi. Molecular Biology Research Center, Baqiyatallah University of Medical Science,Tehran, Iran.Email: fasihi.m@gmail.com

Copyright (C) 2017, IRANIAN JOURNAL OF PATHOLOGY. This is an open-access article distributed under the terms of the Creative Commons Attribution-noncommercia 4.0 International License which permits copy and redistribute the material just in noncommercial usages, provided the original work is properly cited.

\section{Introduction}

Platelet-rich plasma (PRP) constitutes a high concentration of platelets in a solution composed of autologous blood in a small volume of plasma (1). PRP contains $4 \%$ red blood cells (RBCs), 95\% platelets, and $1 \%$ white blood cells (WBCs) (2). Approximately 2 decades ago, it was discovered that one of the major functions of platelets is the secretion of growth factors (3). $\alpha$-Granules available in the platelets contain a high concentration of growth factors such as platelet-derived growth factor (PDGF)-AA, -BB, -AB, platelet-derived angiogenesis factor (PDAF), fibroblast growth factor (FGF), transforming growth factor (TGF)-b1 and $\mathrm{b}-2$, Insulin like growth factor-1 (IGF-1), connective tissue growth factor (CTGF), platelet derived epidermal growth factor (PDEGF), platelet factor-4 (pf-4), vascular endothelial growth factor (VEGF), and epidermal growth factor (EGF) $(4,5)$. As a direct repercussion of the above, the presence of platelets is responsible to accelerate endothelial, epithelial, and epidermal regeneration as well as stimulation of angiogenesis and increase in collagen synthesis (6). It also promotes soft tissue healing, reduces dermal scarring, and enhances homeostatic 
response to injury (7). In addition to growth factors, platelets also release several bioactive proteins such as vitronectin, thrombospondin, and fibronectin. These proteins are responsible to attract macrophages, mesenchymal stem cells, and osteoblasts. Additionally, they also promote the removal of degenerated tissue while enhancing tissue regeneration and healing (8). Since early 2009, PRP therapy is regarded as a popular and natural alternative to surgery since it is a safe and nonsurgical form of biological treatment (3). PRP has attracted tremendous attention within scientific circles and its utility has diversified to a variety of clinical applications such as orthopedics, sports medicine, dentistry, otolaryngology, neurosurgery, ophthalmology, urology, wound healing, cosmetics, cardiothoracic, and maxillofacial surgeries (9). Since PRP is an autologous blood product, it carries no risk of transmissible diseases (10). Platelets are known to play numerous roles in the antimicrobial immune system; they are responsible to produce various oxygen metabolites such as hydrogen peroxide, superoxide, and hydroxyl free radicals (11). Furthermore, they also have a direct effect on the clearance of microorganisms from the blood stream $(12,13)$. Platelets release several peptides known to have antibacterial effects. Examples include fibrinopeptide A, fibrinopeptide B, thymosin $\beta-4$, platelet basic protein, connectivetissue-activating peptide 3, RANTES (regulated on activation, normal T-Cell expressed and secreted), and platelet factor 4 (14).

Although PRP is used in a wide variety of applications, the impact of its intrinsic antibacterial activity on bacteria remains clearly elucidated. To add to the importance of the above statement, the incidence of infections in health care centers remains an important challenge in reconstructive surgery. Therefore, in an attempt to prove the correlation between platelet accumulation and PRP antibacterial activity, in the current study, in vitro assays were used to evaluate and compare PRP antibacterial activity with that of 3 blood products as well as sensitive antibiotic disks.

\section{Materials and Methods
Blood donors and preparation of four products

Venous blood $(20 \mathrm{~mL})$ was collected from 15 healthy males and females between $30-40$ years old. Infection free volunteers who had not consumed any steroidal, antibiotic or anti-inflammatory drugs 7 days prior to the blood collection were selected for this purpose. All volunteers had hemoglobin concentrations higher than $11 \mathrm{~g} / \mathrm{dL}$. It was also compulsory for the volunteers that their platelet numbers be greater than $150 \times 10^{3} / \mathrm{mL}$. Approximately $1 \mathrm{~mL}$ of whole drained blood was separated for baseline whole blood analysis.

Of the individual venous blood samples, $19 \mathrm{~mL}$ was transferred to anticoagulant tubes containing $0.35 \mathrm{~mL}$ of $10 \%$ sodium citrate. The samples were initially centrifuged at $160 \times \mathrm{g}$ for 10 minutes at room temperature. Subsequent to the first centrifugation, 2 phases were observed in each sample: (a) a red lower phase composed of red blood cells and, (b) an upper straw-yellow phase containing the plasma component. The upper surface of the red blood cells, also referred to as the buffy coat, is rich in platelets and leukocytes. Plasma and buffy coat were pipetted with great care and transferred to sterile centrifuge tubes. The plasma and buffy coat were, then, centrifuged for a 2 nd round at $400 \times \mathrm{g}$ for 15 minutes (15). Two layers were eventually seen to form: the upper two-thirds of the sample were designated as platelet-poor plasma (PPP) and the lower one-third was the PRP. The PPP layer was carefully pipetted with a sampler and transferred to a new sterile tube. From each specimen, a $200 \mu \mathrm{L}$ isolate was used to calculate the platelet counts with a Neubauer chamber. Smears were also prepared to evaluate morphology of the platelets.

\section{Evaluation of antimicrobial activity}

Staphylococcus aureus, E. coli, K. pneumonia, Ps. aeruginosa, S. agalactiae, S. epidermidis, Shigella, sp. and Serratia sp. were provided by the Microbiological Laboratory of Clinic Detection Center of IRAN (Tehran, IRAN). 


\section{Agar-Well Diffusion}

Agar-well diffusion method by Mueller-Hinton agar was used to conduct the susceptibility tests (Merck Co., Germany). Four 4-mm diameter wells were punched into the Mueller-Hinton agar plates and a $50-\mu \mathrm{L}$ aliquot of each product was dispensed into the wells. Each product contained PRP produced from the 2-stage centrifugation (PRP-2), PRP obtained from the one-stage centrifugation (PRP-1), PPP, and whole blood (WB).

\section{Disk Diffusion}

The eight bacteria enumerated above were analyzed for susceptibility to penicillin (10 U) and chloramphenicol (30 $\mu \mathrm{g})$ (Oxoid Company) using the Kirby-Bauer disk diffusion method. Guidelines outlined by the Clinical and Laboratory Standards Institute (CLSI) were strictly adhered to for this protocol.

\section{Minimum Inhibitory Concentration}

Minimum inhibitory concentrations (MICs) were determined by the broth microdilution method using commercially available 96-well microtiter plates. Each microtiter plate included positive controls (bacteria without an antimicrobial products), negative controls (Mueller-Hinton medium only), and twofold serial dilutions of each of the 4 antimicrobial products.

To investigate the MIC values, bacteria were first cultivated in Mueller-Hinton broth subsequent to which they were incubated at $37^{\circ} \mathrm{C}$ to quantify susceptibility to the 4 above-mentioned products. After 24 hours, bacterial growth was observed in the logarithmic phase and bacterial turbidity was measured at $600 \mathrm{~nm}$ to determine concentration. Mueller-Hinton broth was then poured into all 96 wells of the microplate. In the microdilution technique, the products in the 1st well were diluted by $10 \%$ and the last well was considered as the control, since no product was added to it. Subsequently, the constant amount of a bacterial culture was added to each well and microplates were incubated at $37^{\circ} \mathrm{C}$ for 24 hours. The following ATCC strains were used as control: Staphylococcus aureus ATCC 25923, Escherichia coli ATCC 25922, Klebsiella pneumonia ATCC 13883, Pseudomonas aeruginosa ATCC 27853,
Streptococcus agalactiae ATCC 12386, Staphylococcus epidermidis ATCC 12228, Shigella sp. ATCC 12022, and Serratia sp. ATCC 39006.

\section{Statistical analysis}

Statistical analysis was accomplished using the SPSS 22.0 (SPSS Inc., Chicago, IL) software. Data in each table were expressed in terms of mean \pm standard deviation. $\mathrm{P}<0.05$ was considered as level of significance.

\section{Ethical approval}

The current study was conducted by the institutional review board of the Baqiyatallah Medical University, Tehran, Iran, in accordance with the Helsinki declaration. The results of the current study were considered confidential and no extra cost was inflicted onto the participants. Written informed consent was obtained from all the study participants; and the volunteers' anonymity was ensured by assigning code numbers to the samples.

\section{Results}

The Prior to fabrication of the products, a CBC test was conducted on each of the 15 volunteers to ensure that the number of present blood cells was within the normal range. In all the smears, it was observed that platelets retained their original normal morphology and aggregation capabilities. Platelets were diluted using the Rees Ecker solution and a Neubauer chamber was employed to compute the platelet count. The average number of platelets in WB, PPP, PRP-1, and PRP-2 were computed as $235908 \pm 38582,25249 \pm 3930,496187 \pm 51823$, and $1193106 \pm 179797$ platelets $/ \mu \mathrm{L}$, respectively.

\section{Results of the Well and Disk Diffusion techniques}

The inhibition zone of bacterial growth was measured for each well on the culture medium. It was observed that in all the plates, there was robust bacterial growth around the wells containing whole blood and platelet-poor plasma. Interestingly, no growth was observed around the wells containing PRP-1 and PRP-2 for Shigella sp., E. coli, S. aureus, $S$. agalactiae, and $S$. epidermidis. However, the inhibition zone for bacterial growth in case of PRP2 was greater than that of PRP-1 under all experimental conditions. As expected, the presence 
of the penicillin disk prevented the growth of Grampositive bacteria and its inhibitory zone was comparable to that of PRP-2. Chloramphenicol disk prevented the growth of all bacteria except $P$. aeruginosa and its inhibitory zones were larger than all experimental samples except for PRP-2 (Table $1)$.

Table 1. Zones of Inhibition

\begin{tabular}{|c|c|c|c|c|c|c|c|c|}
\hline Blood Product and sensitive disk & 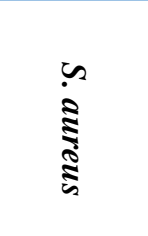 & $\begin{array}{l}-1 \\
\vdots \\
\vdots\end{array}$ & 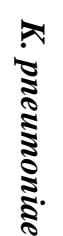 & 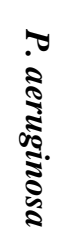 & 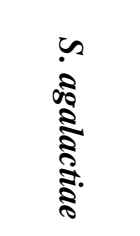 & 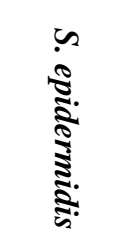 & 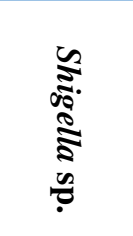 & 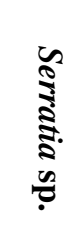 \\
\hline Whole blood & 4 & 4 & 4 & 4 & 4 & 4 & 4 & 4 \\
\hline PRP1 & $10.5 \pm 0.8$ & $9.5 \pm 0.7$ & 4 & 4 & $6.4 \pm 0.8$ & $7.1 \pm 0.7$ & $9.2 \pm 0.9$ & 4 \\
\hline PPP & 4 & 4 & 4 & 4 & 4 & 4 & 4 & 4 \\
\hline PRP2 & $16 \pm 1.3$ & $14.1 \pm 0.6$ & 4 & 4 & $10.2 \pm 1.2$ & $11.5 \pm 1$ & $12.3 \pm 0.8$ & 4 \\
\hline Penicillin disk & 15 & 4 & 4 & 4 & 12 & 13 & 4 & 4 \\
\hline Florfenicol disk & 17 & 16 & 16 & 4 & 15 & 27 & 15 & 17 \\
\hline
\end{tabular}

Table 2. Antibacterial Effect of PRP-2 in Different Concentrations of Platelets

\begin{tabular}{lcccccc}
\hline Name of Bacteria & S. aureus & E. coli & K. pneumoniae & Serratia sp. & P. aeruginosa \\
\hline MIC (platelets $/ \boldsymbol{\mu L})$ & $59655.3 \pm 8989.8$ & $119310.7 \pm 17979.7$ & & & \\
\hline Name of Bacteria & P. aeruginosa & S. agalactiae & S. epidermidis & Shigella sp. & \\
\hline MIC (platelets $/ \boldsymbol{\mu L})$ & & $59655.3 \pm 8989.8$ & $59655.3 \pm 8989.8$ & $119310.7 \pm 17979.7$ & \\
\hline
\end{tabular}

\section{MIC results}

In the current study, the MIC was defined as the lowest platelet concentration that could inhibit bacterial growth. After 24 hours of incubation, the microplates were analyzed to determine the MIC of the products. Bacterial growth was observed in all wells that contained WB, PPP or PRP-1. Based on the results, it was inferred that, in the concentration prepared for the purpose of the current study, these 3 products had no remarkable antibacterial activity against any bacteria. However, it was observed that bacterial growth rate in wells containing PRP-1 was less than those of WB and PPP. PRP-2 at 10\% concentration was observed to inhibit the growth of Shigella ep. and E. coli; at 5\% it inhibited the growth of S. aureus, S. agalactiae, and S. epidermidis; no such effect was observed for $K$. pneumoniae, $P$. Aeruginosa, and Serratia sp. (Table 2).

\section{Minimum Bactericidal Concentration} results

In the current study, minimum bactericidal concentration (MBC) was defined as the minimum product concentration required for killing 99.9\% of all bacteria. PRP-2 was unable to cause bacterial death in any of the wells; it was inferred from the fact that bacterial growth resumed upon transferring to a fresh nutrient culture medium.

\section{Discussion}

Bacterial infection is one of the major challenges that impede and impair tissue healing (16). Controlling post-surgical infections has serious clinical and economic advantages, since it has the potential to drastically reduce the use of antibiotics as well as their associated side effects.

In an attempt to evaluate the effect of the number of platelets on inhibition of bacterial growth, the current study compared the effect of PRP with those of WB, PPP and PRP-1 wherein the platelet concentration was 5, 47 and 2.4 times less than that of found in PRP-2, respectively. The current study results clearly demonstrated that the number of platelets had a significant effect on the inhibition of bacterial growth. The results of the agar-well 
diffusion and MIC assays revealed that WB and PPP had no demonstrable activity against bacterial growth. Although it was observed that PRP-1 was able to reduce the growth of some bacteria, it could not completely prevent the growth of any of the bacteria. The ability of PRP-2 to inhibit the growth of some bacteria can be attributed to the fact that it has the highest number of platelets. It is evident that preparation protocols can significantly influence the number of platelets, hence it can be confidently concluded that the protocol employed to prepare PRP plays an important role in defining its impact on bacterial growth inhibition. Authors understanding and knowledge of the field led them to propose that several fundamental factors had the capability to influence both quantity and quality of PRP. These factors include: (a) the force of gravity $(\mathrm{g})$, (b) the type of the anticoagulant used, (c) the number of centrifugation steps, (d) the type of clotting and activating agent used, (e) the number of platelets originally present in the donor's blood and PRP, and (f) the results of clinical applications (17).

Increment in $g$ can cause an increase in platelet concentrations (18). However, it should be noted that an excessive increase in $g$ during the 1 st centrifugation step can cause the platelets in the buffy coat to form a layer very close to the red blood cell layer. As a result, the quantity and efficacy of PRP can reduce, since during the process of pipetting the buffy coat, there is a risk of either pipetting a large number of the red blood cells or not pipetting a significant portion of the platelet. An increase in $g$ during the 2 nd centrifugation step will cause premature release of the growth factors and have a negative impact on platelet morphology (19). Keeping these considerations and risk factors in mind, the maximum $g$ used in the current research was $\times 400$.

The type of anticoagulant used during the preparation process of PRP also has a particular significant impact (20). The current study used anticoagulant tubes containing sodium citrate. It was done in accordance to previous studies, where it was determined that during the preparation of PRP, ethylene-diamine-tetra-acetic acid (EDTA) causes more platelet damage compared to citrate $(21,22)$. Some recently conducted studies suggested that citrate should be considered as the anticoagulant of choice to prepare PRP for in vivo applications (2325).

It is mandatory that "therapeutic PRP" should have a count of approximately 1 million platelets per microliter in the case of humans (26). In more precise terms, to designate PRP as "therapeutic PRP", it should have a platelet concentration of at least $300 \%$ to $400 \%$ higher than that of the whole blood (27). The 2-step centrifugation technique is applied to obtain the required number of platelets in PRP $(17,28)$. The singlestep, on the other hand, produces a mixture of PRP and PPP in such a way that a low platelet concentration is obtained. In the current study, the platelet compositions of PRP-1 and PRP-2 were 2 and 5 times more than that of the whole blood, respectively. This difference in platelet number impacted the antibacterial activity, resulting in a variation in the effects of these 2 products (29).

The current study evaluated the effect of PRP preparation protocol on the quality and quantity of platelets through platelet smear analysis. It was done since it is a well-established fact that the protocol used to prepare PRP can inflict damage on platelets $(30,31)$. Officially, the results of the smear analysis were accepted for the purpose of downstream analysis of the parameters of platelet function such as changes in morphology, size, staining specifications, degree of activation, and clump formation.

Many researchers believe that the presence of leukocytes in PRP affects bacterial growth as a result of myeloperoxidase release. However, it is demonstrated that while the concentrations of myeloperoxidase in PRP and PPP are similar, their antibacterial activities are significantly different. It is indicated that the robust antibacterial activity of PRP is directly related to the presence of platelets and has no correlation with the presence of white blood cells. Drago et al., proved this hypothesis by selectively removing leukocytes for the purpose of producing a pure version of PRP (32).

The current study used the agar-well diffusion technique to perceive that while PRP-1 and PRP-2 inhibited the growth of Shigella sp., E. coli, S. 
aureus, S. agalactiae, and S. epidermidis, they had no effect on the growth of $K$. pneumoniae, $P$. aeruginosa, and Serratia sp. (30). Bielecki et al., also used the agar-well diffusion technique to study the PRP mediated antibacterial activity. Their results also indicated that PRP inhibited the growth of $S$. aureus and E. coli, but had no effect on the growth of $K$. pneumoniae, E. faecalis, and $P$. aeruginosa. The results of the current study corroborated the results obtained by the above mentioned study. The maximum antibacterial effect of PRP was observed 2-8 hours after the cultivation of bacteria (30).

In the current study, the antibacterial activity of the platelets was compared to those of chloramphenicol and penicillin antibacterial sensitive disks. In all cases where PRP-2 was observed to prevent bacteria growth, the effect of the chloramphenicol disk was more than that of PRP-2. The effect of the penicillin disk on the growth of Gram-positive bacteria was almost the same as that of PRP-2. The effect of PRP-1, however, was uniformly less than those of chloramphenicol and penicillin disks in all cases.

The MIC results generated in the current study were in line with those of Drago et al. who claimed that PRP-2 inhibited the growth of certain bacteria at $5 \%$ and $10 \%$ concentrations. The only significant difference between the 2 studies was in terms of the number of platelets per microliter required for bacteria growth inhibition, which in the current case was far removed from those determined by the above mentioned study (32).

Although the antibacterial effect of PRP is observed and described by several published studies, the exact mechanism underlying the process is not completely understood and remains elucidated $(30,32)$. It is hypothesized that to prevent the growth of bacteria, platelets release several proteins and peptides that possess antibacterial activity; additionally, they also have the capacity to generate oxygen metabolites $(13,33)$.

Platelet $\alpha$-granules contain not only growth factors and antimicrobial peptides, but also catecholamines, serotonin, osteonectin, proaccelerin, vitronectin, thrombospondin, and fibronectin along with several other substances (28). These compounds are released when platelets are present at a very high concentration. The release of all these compounds and chemicals helps to prevent bacterial growth and accelerate tissue healing (34).

The current study was conducted to assess the inhibitory activities of PRP and PPP on the growth of commonly prevalent pathogenic bacteria in Iran. E. coli (35-38), K. pneumonia (39-41), P. aeruginosa (42-43), Staphylococcus spp. (44-45), Serratia spp. (46), and Shigella spp.(47-50) infections are prevalent in Iran and their resistance to the most frequently used antibiotics is reported. The current study finding showed reasonable in vitro inhibitory activities of PRP and PPP on the growth of these pathogenic bacteria.

\section{Conclusion}

According to the results of the current study, it can be summarized that PRP has a significant antibacterial activity. In addition, via the agar-well diffusion and MIC methodologies, it is now proved that the number of platelets or concentration of the PRP is directly proportional to the antibacterial activity.

Conflict of Interest: The authors declare that there was no conflict of interest.

\section{References}

1. Dhurat R, Sukesh M. Principles and Methods of Preparation of Platelet-Rich Plasma: A Review and Author's Perspective. J Cutan Aesthet Surg. 2014;7(4):189-197.

2. Narang I, Mittal N, Mishra N. A comparative evaluation of the blood clot, platelet-rich plasma, and platelet-rich fibrin in regeneration of necrotic immature permanent teeth: A clinical study. Contemp Clin Dent. 2015;6(1):63-68.

3. Dhillon RS, Schwarz EM, Maloney MD. Platelet-rich plasma therapy - future or trend? Arthritis Res Ther. 2012;14(4):219.

4. Lubkowska A, Dolegowska B, Banfi G. Growth factor content in PRP and their applicability in medicine. J Biol Regul Homeost Agents. 2012;26(2 Suppl 1):3S-22S. 
5. Blair P, Flaumenhaft R. Platelet alphagranules: basic biology and clinical correlates. Blood Rev. 2009;23(4):177-189.

6. Middleton KK, Barro V, Muller B, Terada S, Fu FH. Evaluation of the effects of platelet-rich plasma (PRP) therapy involved in the healing of sports-related soft tissue injuries. Iowa Orthop J. 2012;32:150-163.

7. Guo S, Dipietro LA. Factors affecting wound healing. J Dent Res. 2010;89(3):219-229.

8. Civinini R, Macera A, Nistri L, Redl B, Innocenti $\mathrm{M}$. The use of autologous bloodderived growth factors in bone regeneration. Clin Cases Miner Bone Metab. 2011;8(1):25-31.

9. Sampson S, Gerhardt M, Mandelbaum B. Platelet rich plasma injection grafts for musculoskeletal injuries: a review. Curr Rev Musculoskelet Med. 2008;1(3-4):165-174.

10. Kasten P, Vogel J, Geiger F, Niemeyer $\mathrm{P}$, Luginbuhl R, Szalay K. The effect of plateletrich plasma on healing in critical-size long-bone defects. Biomaterials. 2008;29(29):3983-3992.

11.Xie X, Zhang C, Tuan RS. Biology of platelet-rich plasma and its clinical application in cartilage repair. Arthritis Res Ther. 2014;16(1):204.

12. Weksler BB, Nachman RL. Rabbit platelet bactericidal protein. J Exp Med. 1971;134(5):1114-1130.

13. Krijgsveld J, Zaat SA, Meeldijk J, van Veelen PA, Fang G, Poolman B, et al. Thrombocidins, microbicidal proteins from human blood platelets, are C-terminal deletion products of CXC chemokines. J Biol Chem. 2000;275(27):20374-20381.

14. Tang YQ, Yeaman MR, Selsted ME. Antimicrobial peptides from human platelets. Infect Immun. 2002;70(12):6524-6533.

15. Maghsoudi O, Beheshtiha SHS, Abarkar M, Anvar SA. Standardization and Modification Techniques of Platelet-Rich Plasma (PRP) Preparation in Rabbit. ICPJ. 2015;1(2):1-5.

16. Demidova-Rice TN, Hamblin MR, Herman IM. Acute and impaired wound healing: pathophysiology and current methods for drug delivery, part 1: normal and chronic wounds: biology, causes, and approaches to care. Adv Skin Wound Care. 2012;25(7):304-314.

17. Nagata MJ, Messora MR, Furlaneto FA, Fucini SE, Bosco AF, Garcia VG, et al.
Effectiveness of two methods for preparation of autologous platelet-rich plasma: an experimental study in rabbits. Eur J Dent. 2010;4(4):395-402.

18. Marx RE, Carlson ER, Eichstaedt RM, Schimmele SR, Strauss JE, Georgeff KR. Platelet-rich plasma: Growth factor enhancement for bone grafts. Oral Surg Oral Med Oral Pathol Oral Radiol Endod. 1998;85(6):638-646.

19. Dugrillon A, Eichler H, Kern S, Kluter H. Autologous concentrated platelet-rich plasma (cPRP) for local application in bone regeneration. Int $\mathrm{J}$ Oral Maxillofac Surg. 2002;31(6):615-619.

20. Marx RE. Platelet-rich plasma (PRP): what is PRP and what is not PRP? Implant Dent. 2001;10(4):225-228.

21. Efeoglu C, Akcay YD, Erturk S. A modified method for preparing platelet-rich plasma: an experimental study. J Oral Maxillofac Surg. 2004;62(11):1403-1407.

22. Macey M, Azam U, McCarthy D, Webb L, Chapman ES, Okrongly D, et al. Evaluation of the anticoagulants EDTA and citrate, theophylline, adenosine, and dipyridamole (CTAD) for assessing platelet activation on the ADVIA 120 hematology system. Clin Chem. 2002;48(6 Pt 1):891-899.

23.Lozada JL, Caplanis N, Proussaefs P, Willardsen J, Kammeyer G. Platelet-rich plasma application in sinus graft surgery: Part I-Background and processing techniques. J Oral Implantol. 2001;27(1):38-42.

24. Snyder EL, Calhoun BC. Topical platelet growth factor therapy: of lotions and potions. Transfusion. 2001;41(10):1186-1189.

25.Zimmermann R, Jakubietz R, Jakubietz M, Strasser E, Schlegel A, Wiltfang J, et al. Different preparation methods to obtain platelet components as a source of growth factors for local application. Transfusion. 2001;41(10):1217-1224.

26. Kececi Y, Ozsu S, Bilgir O. A costeffective method for obtaining standard plateletrich plasma. Wounds. 2014;26(8):232-238.

27. Marx RE. Platelet-rich plasma: evidence to support its use. J Oral Maxillofac Surg. 2004;62(4):489-496.

28. Anitua E, Andia I, Sanchez M, Azofra J, del Mar Zalduendo M, de la Fuente M, et al. Autologous preparations rich in growth factors 
promote proliferation and induce VEGF and HGF production by human tendon cells in culture. J Orthop Res. 2005;23(2):281-286.

29. Halmay D, Sotonyi P, Vajdovich P, Gaal T. Morphological evaluation of canine platelets on Giemsa- and PAS-stained blood smears. Acta Vet Hung. 2005;53(3):337-350.

30. Bielecki TM, Gazdzik TS, Arendt J, Szczepanski T, Krol W, Wielkoszynski T. Antibacterial effect of autologous platelet gel enriched with growth factors and other active substances: an in vitro study. J Bone Joint Surg Br. 2007;89(3):417-420.

31. Dohan DM, Choukroun J, Diss A, Dohan SL, Dohan AJ, Mouhyi J, et al. Plateletrich fibrin (PRF): a second-generation platelet concentrate. Part III: leucocyte activation: a new feature for platelet concentrates? Oral Surg Oral Med Oral Pathol Oral Radiol Endod. 2006;101(3):e51-55.

32. Drago L, Bortolin M, Vassena C, Taschieri S, Del Fabbro M. Antimicrobial activity of pure platelet-rich plasma against microorganisms isolated from oral cavity. BMC Microbiol. 2013;13(1):47.

33. Kraemer BF, Campbell RA, Schwertz H, Cody MJ, Franks Z, Tolley ND, et al. Novel antibacterial activities of beta-defensin 1 in human platelets: suppression of pathogen growth and signaling of neutrophil extracellular trap formation. PLoS Pathog. 2011;7(11):e1002355.

34. Tohidnezhad M, Varoga D, Wruck CJ, Brandenburg LO, Seekamp A, Shakibaei M, et al. Platelet-released growth factors can accelerate tenocyte proliferation and activate the anti-oxidant response element. Histochem Cell Biol. 2011;135(5):453-460.

35. Jahandeh N, Ranjbar R, Behzadi P, Behzadi E. Uropathogenic Escherichia coli virulence genes: invaluable approaches for designing DNA microarray probes. Cent European J Urol. 2015;68(4):452-8.

36. Tajbakhsh E, Khamesipour F, Ranjbar R, Ugwu IC. Prevalence of class 1 and 2 integrons in multi-drug resistant Escherichia coli isolated from aquaculture water in Chaharmahal Va Bakhtiari province, Iran. Ann Clin Microbiol Antimicrob. $2015 ; 14: 37$.

37. Farshad S, Ranjbar R, Japoni A, Hosseini M, Anvarinejad M, Mohammadzadegan R. Microbial susceptibility, virulence factors, and plasmid profiles of uropathogenic Escherichia coli strains isolated from children in Jahrom, Iran. Arch Iran Med. 2012 May;15(5):312-6

38. Momtaz H, Karimian A, Madani M, Safarpoor Dehkordi F, Ranjbar R, Sarshar M, Souod N. Uropathogenic Escherichia coli in Iran: serogroup distributions, virulence factors and antimicrobial resistance properties. Ann Clin Microbiol Antimicrob. $2013 ; 12: 8$.

39. Ranjbar R, Memariani H, Sorouri R, Memariani M. Distribution of virulence genes and genotyping of CTX-M-15-producing Klebsiella pneumoniae isolated from patients with community-acquired urinary tract infection (CA-UTI). Microb Pathog. 2016 ;100:244-249.

40. Ghafourian S, Bin Sekawi Z, Sadeghifard N, Mohebi R, Kumari Neela V, Maleki A, et al. The Prevalence of ESBLs Producing Klebsiella pneumoniae Isolates in Some Major Hospitals, Iran. Open Microbiol J. 2011;5:91-5.

41. Ranjbar R, Memariani H, Sorouri R, Memariani M. Distribution of virulence genes and genotyping of CTX-M-15-producing Klebsiella pneumoniae isolated from patients with community-acquired urinary tract infection (CA-UTI). Microb Pathog. 2016 ;100:244-249.

42. Ranjbar R, Owlia P, Saderi H, Bameri Z, Izadi M, Jonaidi N,et al. Isolation of clinical strains of Pseudomonas aeruginosa harboring different plasmids. Pak J Biol Sci. 2007 ;10(17):3020-2.

43. Ranjbar R, Owlia P, Saderi H, Mansouri $S$, Jonaidi-Jafari $N$, Izadi $M$, et al. Characterization of Pseudomonas aeruginosa strains isolated from burned patients hospitalized in a major burn center in Tehran, Iran. Acta Med Iran. 2011;49(10):675-9.

44. Panahi Y, Sattari M, Pour Babaie A, Beiraghdar F, Ranjbar R, Hedaiat Joo A, et al. The Essential Oils Activity of Eucalyptus polycarpa, E. largiflorence, E. malliodora and E. camaldulensis on Staphylococcus aureus. Iran J Pharm Res. 2011 Winter;10(1):43-8.

45. Ranjbar R, Takhtfooladi MA. The effects of photobiomodulation therapy on Staphylococcus aureus infected surgical wounds in diabetic rats. A microbiological, histopathological, and biomechanical study. Acta Cir Bras. 2016 ;31(8):498-504. 
46. Mostatabi N, Farshad S, Ranjbar R. Molecular evaluations of extended spectrum $\beta$ lactamase producing strains of Serratia isolated from blood samples of the patients in Namazi Hospital, Shiraz, Southern Iran.Iran J Microbiol. 2013 ;5(4):328-33.

47. Ranjbar R, Soltan-Dallal MM, Pourshafie MR, Mammina C. Antibiotic resistance among Shigella serogroups isolated in Tehran, Iran (2002-2004). J Infect Dev Ctries. 2009 ;3(8):647-8.

48. Ranjbar R, Hosseini MJ, Kaffashian AR, Farshad S. An outbreak of shigellosis due to
Shigella flexneri serotype $3 \mathrm{a}$ in a prison in Iran. Arch Iran Med. 2010 ;13(5):413-6.

49. Filliol-Toutain I, Chiou CS, Mammina C, Gerner-Smidt P, Thong KL, Phung DC, Pichel M, et al. Global Distribution of Shigella sonnei Clones. Emerg Infect Dis. 2011 ;17(10):1910-2.

50. Ranjbar R, Aleo A, Giammanco GM, Dionisi AM, Sadeghifard N, Mammina C. Genetic relatedness among isolates of Shigella sonnei carrying class 2 integrons in Tehran, Iran, 2002-2003. BMC Infect Dis. 2007;22;7:62.

\section{How to Cite This Article:}

Maghsoudi O, Ranjbar R, Mirjalili SH, Fasihi Ramandi M. Inhibitory activities of platelet-rich and plateletpoor plasma on the growth- of pathogenic bacteria. Iranian Journal of Pathology. 2017; 12(1): 79-87 